\title{
Triangulating Unknown Environments using Robot Swarms
}

\author{
Aaron Becker \\ Computer Science \\ Rice University \\ USA
Seoung Kyou Lee
Computer Science
Rice University
USA

\author{
Sándor P. Fekete \\ Computer Science \\ TU Braunschweig \\ Germany
}

\author{
James McLurkin \\ Computer Science \\ Rice University \\ USA
}

\author{
Alexander Kröller \\ Computer Science \\ TU Braunschweig \\ Germany
}

\section{Christiane Schmidt \\ Computer Science \\ TU Braunschweig \\ Germany}

\section{Categories and Subject Descriptors}

F.2.2 [Nonnumerical Algorithms and Problems]: Geometrical problems and computations; I.2.0 [Robotics]: Autonomous vehicles

\section{General Terms}

Algorithms

\section{Keywords}

Online optimization; triangulation; exploration and searching; robot navigation; robot swarms; r-one robots

\section{INTRODUCTION}

In recent years, the field of robotics has seen two diverging trends. One has been to achieve progress by increasing the capabilities of individual robots, keeping the cost of state-of-art machines relatively high. An opposite direction has been to develop simpler and cheaper platforms, at the expense of reducing the capabilities per robot. The latter raises new challenges for developing new principles and algorithms, such as coordinating many robots with limited capabilities into a swarm that can carry out difficult tasks, for example, exploration, surveillance, and guidance.

In this video, we show a recent collaboration between theory and practice of swarm robotics. We consider online problems related to exploring and surveying a region by a swarm of robots with limited communication range. The minimum relay triangulation problem (MRTP) asks for placing a minimum number of robots, such that their communication graph is a triangulated cover of the region. The maximum area triangulation problem (MATP) aims at finding a placement of $n$ robots such that their communication graph contains a root and forms a triangulated cover of a maximum possible amount of area. We demonstrate the practical relevance of our methods by showing how they can be used on the novel real-world platform $r$-one.

Copyright is held by the author/owner(s).

SoCG'13, June 17-20, 2013, Rio de Janeiro, Brazil.

ACM 978-1-4503-2031-3/13/06.

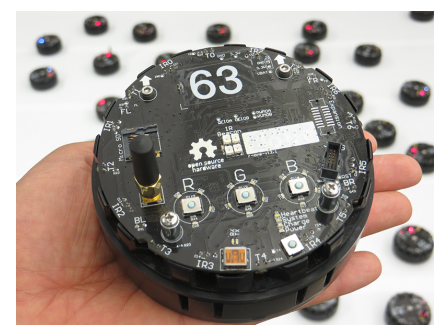

(a) r-one robot

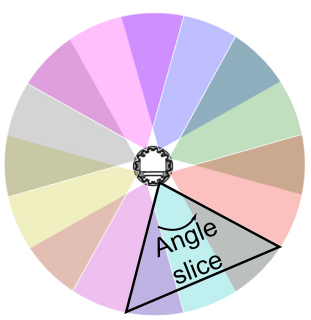

(b) IR regions
Figure 1: (a) The r-one for multi-robot research, designed by the MRSL group at Rice University. (b) IR receiver detection regions. Each receiver detects an overlapping $68^{\circ}$, allowing to determine angles within $22.5^{\circ}$.

\section{THEORETICAL MODEL}

We are given a polygon $P$. Every robot in the swarm has a (circular) communication range $r$. Within this range, perception of and communication with other robots is possible. For ease of description, we normalize to $r=1$.

In the Minimum Relay Triangulation Problem (MRTP), we are given the $n$-gon $P$ and a point $z \in P$. The goal is to compute a set $R$ (with $z \in R$ and $V \subseteq R$ for the vertex set $V$ of $P$ ) of relays within $P$, such that there is a unit triangulation of $P$ whose vertex set is exactly the set $R$, whose edges stay within $P$ and have length at most 1 . The objective is to minimize the number of relays.

In the Maximum Area Triangulation Problem (MATP), the number of available relays is bounded by a number $k$. The goal is to determine a set $R$ of at most $k$ relays, with a unit triangulation covering the maximum possible area.

For the online versions (OMRTP and OMATP), the polygon $P$ is unknown. Each relay may move through the area, and has to decide on a new location for a triangulation vertex, while still being within reach of the previously placed relays. Once it has stopped, it becomes part of the static triangulation, allowing other relays to extend the exploration.

\section{THE R-ONE ROBOT}

The r-one [5] is an advanced, low-cost, open source robot design with an estimated unit cost of about US $\$ 250$. Mea- 


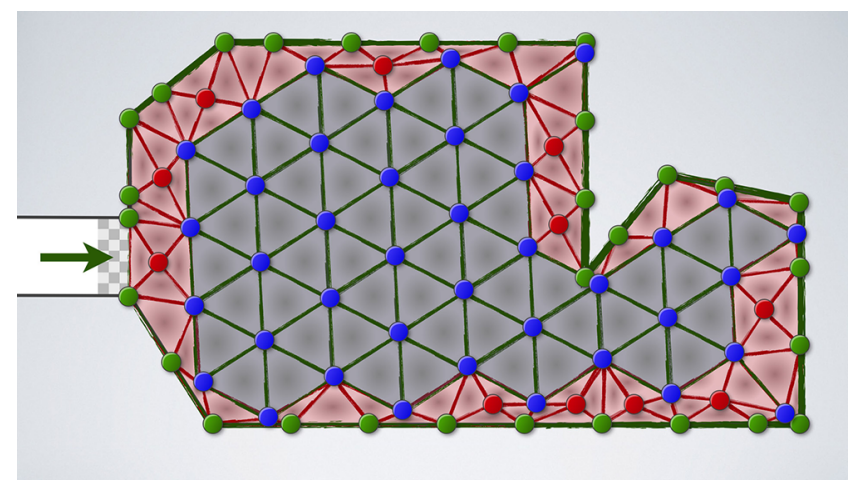

Figure 2: The 3-competitive strategy for the OMRTP.

suring only $11 \mathrm{~cm}$ in diameter, it has a 32-bit ARM-based microcontroller, running at $50 \mathrm{MHz}$ with no floating point unit. The local infrared (IR) communication system is used for inter-robot communication and localization. Each robot has eight IR transmitters and eight receivers. The transmitters broadcast in unison and emit a radially uniform energy pattern. The robot's eight IR receivers are radially spaced to produce 16 distinct detection regions (shown in Figure 1b). By monitoring the overlapping regions, the bearing of neighbors can be estimated to within $\approx \pi / 8$. Thus, it has limited capabilities for measurement, which is intertwined with local communication. The IR receivers have a maximum bit rate of 1250 bits per second. Each robot transmits $(\Delta+1) 4$-byte messages during each round, one being a system announce message, the others containing the bearing measurements to that robot's neighbors. The system supports a maximum of $\Delta=10$. For experimental work on coordination and navigation of r-ones, see [1].

\section{MRTP AND MATP}

The problems MRTP and MATP were introduced in [4]; the currently best results for the online versions OMRTP and OMATP were presented in $[3,6]$. Both problems share their decision problem, which is known to be NP-hard.

For the OMRTP, there is a lower bound of $6 / 5$ on the competitive factor of any deterministic strategy, as well as a 3-competitive algorithm for general polygons. This strategy is shown in Figure 2: We place robots at unit intervals along the boundary (green) and fill the interior with a regular triangular grid (blue). The space between the two is patched together using a third class (red). One can prove that the size each of the three classes is bounded by the number of robots in an optimal solution. For polyominoes, algorithms with better competitive factors exist [2].

On the other hand, the OMATP does not admit a deterministic strategy with a constant competitive factor, if the polygon may have small corridors. If these can be excluded, greedy strategies perform well [2].

\section{THE VIDEO}

The video starts by showing a swarm of robots. Each of them perceives only its direct environment. This leads to the question of exploring an unknown environment, and keeping it under surveillance. The next scene introduces triangulations as a way to build a covering network whose geometry describes the environment. A prototype of of such

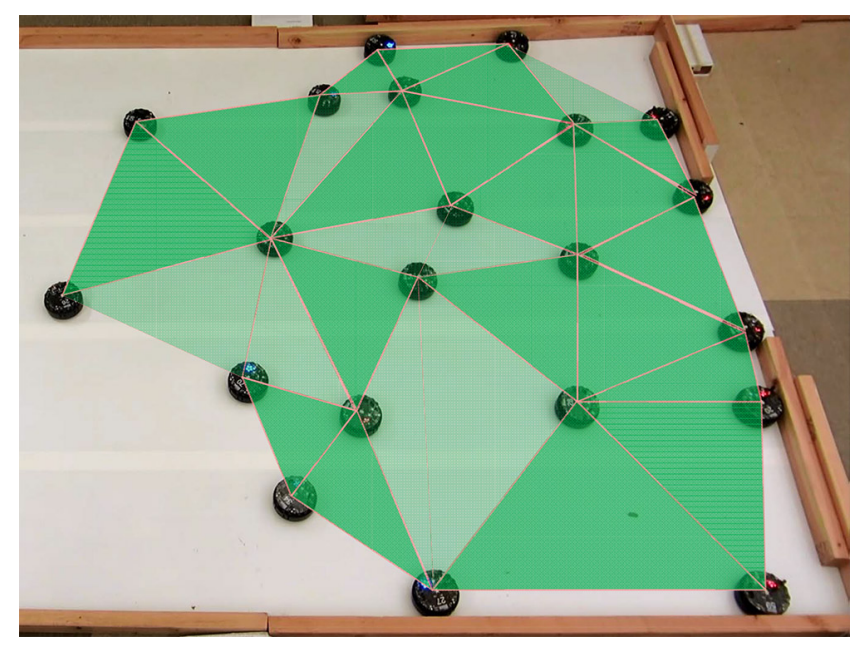

Figure 3: A swarm of r-ones executing the greedy algorithm for the OMATP.

low-cost robots is the r-one platform, which is introduced. This is followed by a theoretical description of the MRTP, for which the 3-competitive algorithm is presented. Then, the MATP is introduced, and a greedy algorithm executed by r-one robots is shown-see Figure 3.

Acknowledgements. We thank Marcus Brandenburger and Henning Hasemann for their support in making this video.

\section{REFERENCES}

[1] A. Cornejo, A. Lynch, E. Fudge, S. Bilstein, M. Khabbazian, and J. McLurkin. Scale-free coordinates for multi-robot systems with bearing-only sensors. In Algorithmic Foundations of Robotics X, volume 86 of Springer Tracts in Advanced Robotics, pages 397-414. Springer, 2013.

[2] S. Fekete, S. Rex, and C. Schmidt. Online exploration and triangulation in orthogonal polygonal regions. In WALCOM: Algorithms and Computation, volume 7748 of Lecture Notes in Computer Science, pages 29-40. Springer Berlin Heidelberg, 2013.

[3] S. P. Fekete, T. Kamphans, A. Kröller, J. S. Mitchell, and C. Schmidt. Exploring and triangulating a region by a swarm of robots. In Proc. 14th. International Workshop on Approximation Algorithms for Combinatorial Optimization Problems, pages 206-217. Springer, 2011.

[4] S. P. Fekete, T. Kamphans, A. Kröller, and C. Schmidt. Robot swarms for exploration and triangulation of unknown environments. In Proceedings of the 25th European Workshop on Computational Geometry, pages 153-156, 2010.

[5] J. McLurkin, A. Lynch, S. Rixner, T. Barr, A. Chou, K. Foster, and S. Bilstein. A low-cost multi-robot system for research, teaching, and outreach. In Distributed Autonomous Robotic Systems, volume 83 of Springer Tracts in Advanced Robotics, pages 597-609. Springer Berlin Heidelberg, 2013.

[6] C. Schmidt. Algorithms for Mobile Agents with Limited Capabilities. Phd thesis, Braunschweig Institute of Technology, 2011. 